2006, 110, 8907-8909

Published on Web 04/18/2006

\title{
Contribution of Omega-3 Fatty Acids to the Thermodynamics of Membrane Protein Solvation
}

\author{
Alan Grossfield, ${ }^{*} \uparrow$ Scott E. Feller,$\stackrel{ }{\ddagger}$ and Michael C. Pitman ${ }^{\dagger}$ \\ IBM T. J. Watson Research Center, 1101 Kitchawan Rd., P.O. Box 218, Yorktown Heights, New York 10598, \\ and Department of Chemistry, Wabash College, 301 West Wabash Avenue, Crawfordsville, Indiana 47933
}

Received: January 19, 2006; In Final Form: March 20, 2006

\begin{abstract}
Recent NMR experiments and molecular dynamics simulations have indicated that rhodopsin is preferentially solvated by omega-3 fatty acids compared to saturated chains. However, to date no physical theory has been advanced to explain this phenomenon. The present work presents a novel thermodynamic explanation for this preferential solvation based on statistical analysis of $26100 \mathrm{~ns}$ all-atom molecular dynamics simulations of rhodopsin in membranes rich in polyunsaturated chains. The results indicate that the preferential solvation by omega- 3 chains is entropically driven; all chains experience an entropic penalty when associating with the protein, but the penalty is significantly larger for saturated chains.
\end{abstract}

Over the past 25 years, a large number of studies have demonstrated the importance of polyunsaturated fatty acids (PUFAs) to human cognitive and visual development. ${ }^{1}$ Similarly, several different experimental techniques and molecular dynamics simulations have demonstrated that membranes rich in PUFAs have physical properties very different from more saturated membranes $;{ }^{2-5}$ polyunsaturated chains are very flexible, leading to low order parameters and short correlation times. These properties in turn modulate the behavior of proteins embedded in them. ${ }^{6-9}$ For example, the photocycle kinetics of rhodopsin, the G-protein coupled receptor (GPCR) responsible for dim-light vision, are greatly enhanced by the presence of docosahexaenoyl (DHA) chains, ${ }^{10}$ while several simulations have demonstrated enrichment of polyunsaturated chains at rhodopsin's surface. ${ }^{11-13}$ Although recent work ${ }^{12}$ has suggested functional implications for this behavior, at present no theory has been advanced to explain the physical origins of this behavior. We present here a statistical analysis of lipid chain conformations accumulated from 26 independently constructed $100 \mathrm{~ns}$ all-atom molecular dynamics simulations, ${ }^{12}$ which suggests a thermodynamic explanation. The results indicate that the phenomenon is entropically driven; all chains pay an entropic penalty to interact with the protein, but the penalty is significantly smaller for DHA than for a saturated chain. Although there is a partially compensating enthalpic effect, the net result is a preference for DHA of roughly $0.5-1.0 k_{\mathrm{B}} T$ per chain.

The raw data for this work was derived from a series of 26 molecular dynamics simulations. Each simulation contained a single rhodopsin molecule embedded in a 99-lipid membrane containing a 2:2:1 molar ratio of 1-stearoyl-2-docosahexaenoylphosphatidylethanolamine, 1-stearoyl-2-docosahexaenoyl-phosphatidylcholine, and cholesterol. Each lipid contained an 18carbon saturated chain (STEA) at the sn-1 position and a 22-

\footnotetext{
* Corresponding author. E-mail: agrossf@us.ibm.com

$\dagger$ IBM.

$\doteqdot$ Wabash College.
}

carbon $\omega$-3 polyunsaturated chain (DHA) at the sn-2 position. All of the simulations were run for roughly $100 \mathrm{~ns}$, with the first $20 \mathrm{~ns}$ discarded as equilibration. Each simulation started with identical protein coordinates, while the membranes were constructed independently using lipid structures taken from a previously generated library (for details see Supporting Information and ref 12).

Figure 1 shows the mole fraction for the three major membrane components (DHA, STEA, and cholesterol), computed as a function of the signed distance to the protein surface (see the Supporting Information for ref 13 for details). The data confirm what was seen in previous simulations: the concentration of DHA is greatly enriched near the protein surface, while the STEA chain is depleted. The mole fraction has been normalized to 1 , while the absolute population of chain atoms is very small at distances less than $-5 \AA$. The depletion of cholesterol at the surface and slight enrichment 5-10 A away are also consistent with previous work.

We begin the thermodynamic analysis by defining a set of discrete states for the lipid chains. For the saturated chains, this was done by classifying each torsion into one of three states (trans, gauche + , and gauche-), resulting in a vocabulary of $3^{14}$ states. Since the vast majority of these states are poorly populated, we chose to aggregate consecutive torsions and defined three new states (both trans, both gauche, and mixed), which reduces the number of states and improves the quality of the statistics. The situation is somewhat more complex for DHA; the double-bonded torsions are locked in a single conformation and thus make no contribution to the chain statistics. The saturated torsions are significantly altered by the presence of neighboring double bonds, and contain only two states (skew + and skew-) with broad minima and a small barrier between them. ${ }^{2,3}$ This set of states is not unique, and other definitions are possible (e.g., via clustering from simulation data). However, the present approach is simple, independent of the force field and simulation conditions and easily transferable 


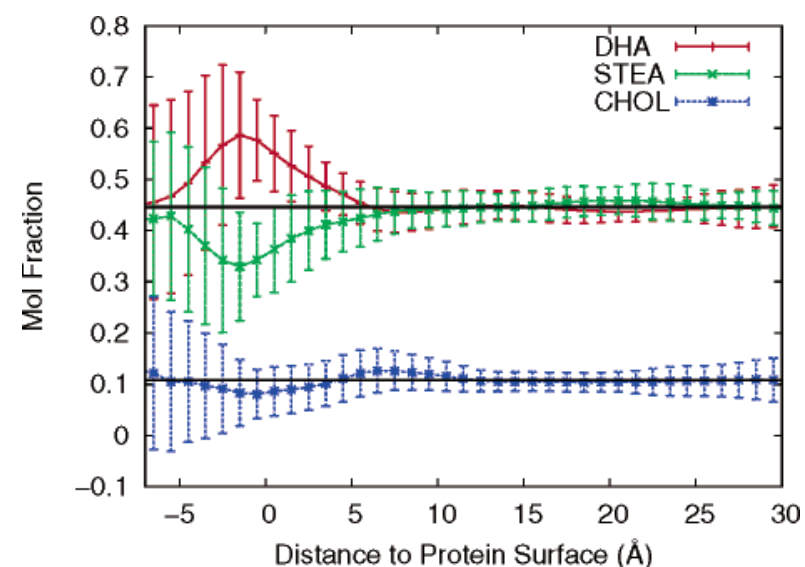

Figure 1. Mole fraction of the membrane components as a function of distance from the protein surface. The mole fraction is computed on an atomic basis. The distance to the surface is the signed distance used previously. ${ }^{13}$ Error bars are the statistical uncertainty, computed as the standard deviation across the individual simulations. Solid black lines are the expected mole fractions for a uniform distribution.

to other lipid species, such as monounsaturated chains. Moreover, the isomeric state approximation has long been used in mean field models of membrane structure, including analyses of structural perturbations due to inclusions such as transmembrane domains. ${ }^{14}$ Given a discrete vocabulary of states $s_{\mathrm{i}}$, it is possible to compute the probability distribution of chain states $P\left(s_{i}\right)$ from a molecular dynamics simulation. We can then compute the Gibbs entropy as

$$
S=-k_{\mathrm{B}} \sum P\left(s_{i}\right) \log P\left(s_{i}\right)
$$

Further, if we also know the probability distribution for the chains in a bulk environment $P^{*}\left(s_{i}\right)$, we can also compute the relative entropy of the two distributions, which was identified by Qian $^{15}$ as the free energy to perturb the distribution

$$
\Delta G=-k_{\mathrm{B}} T \sum P\left(s_{i}\right) \log \frac{P\left(s_{i}\right)}{P^{*}\left(s_{i}\right)}
$$

For purposes of this analysis, the chain state distribution for lipids $16-18 \AA$ from the protein surface was used to represent the unperturbed lipid state. This range was chosen because it is far enough from the protein surface that the lipid isomerization is likely to be relatively unperturbed, but not so far that there are too few lipids for good statistics. The results are not particularly sensitive to the choice of which lipids are used to compute $P^{*}\left(s_{i}\right)$. With the relative free energy and absolute entropy known, we can also compute the relative chain enthalpy up to an arbitrary constant. This constant may not be the same for the two chain species considered here.

The main distinction between previous theoretical approaches and the present work is the role of the distribution of chain states. For example, in most mean field models, this distribution is the primary result, determined by the theory combined with specific boundary conditions (e.g., a hydrophobic cylinder model for a membrane protein), ${ }^{9,14,16-20}$ while in the present work these distributions are determined in advance by explicit all-atom simulations.

The results of this analysis are shown in Figure 2. Part A shows the chain free energy as a function of distance to the protein surface. The free energy curves for both species rise as they approach the protein, but the STEA curve increases by roughly $0.4 \mathrm{kcal} / \mathrm{mol}$ more. Part B shows the entropic contribu-
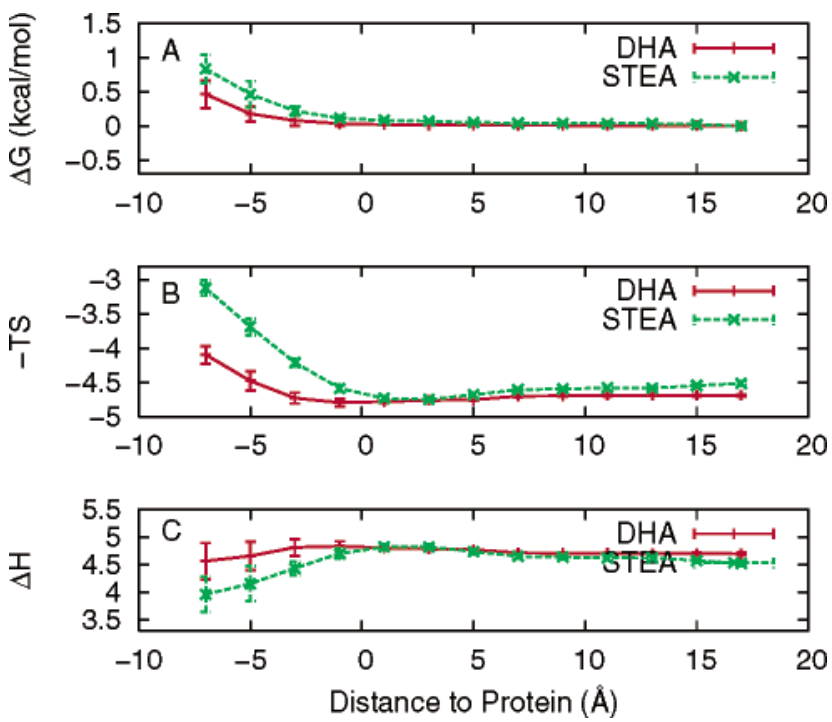

Figure 2. Chain thermodynamics as a function of distance to the protein surface. Part A shows the free energy for the chain, relative to the bulk distribution. Part B shows the absolute chain entropy, multiplied by $-\mathrm{T}$, to make the units $\mathrm{kcal} / \mathrm{mol}$. Part $\mathrm{C}$ shows the relative chain enthalpy, computed by summing the curves from Parts A and B. Error bars are the statistical uncertainties, computed as the standard deviation of the values computed from the individual simulation. The distance to the surface is computed as defined previously.

tions to the free energy; the curves are qualitatively similar, but the differences between STEA and DHA are magnified, indicating that the phenomenon is entropically driven. The relative enthalpy, shown in Part $\mathrm{C}$, behaves differently. The DHA enthalpy is essentially independent (within statistical uncertainty) of its location, while the STEA enthalpy drops somewhat upon association with the protein. Interestingly, the favorable change in enthalpy occurs despite a 5-fold decrease in the probability of the all-trans state, which is the most likely chain state at all distances.

The energetic degeneracy of the skew + and skew - states of DHA means that the energy landscape for DHA is quite flat, while by contrast the trans state of STEA is roughly $0.5 \mathrm{kcal} /$ mol more favorable than the gauche states. This accounts for the difference in the enthalpy curves; since all allowed DHA states are roughly equal in energy, the only mechanism for an enthalpy change would be differences in DHA environment packing interactions as reflected by changes in the chain statistics. By contrast, a small increase in the number of trans torsions (roughly 1 per chain) or decrease in intrachain strain would be sufficient to explain STEA's enthalpy changes. It is important to note there are other possible sources of enthalpy differences, e.g., variations in protein-headgroup interactions. These factors contribute to the probability distributions observed in the molecular dynamics simulations and thus, in principle, are implicitly captured as the effective enthalpies of various chain states. However, some information may be lost in the preaveraging inherent in the definitions of the chain states, such as variations in energy due to overall lipid rotation. Unlike other theoretical approaches, where the chain enthalpies are directly specified by the model, the present approach treats chain enthalpy as a derived quantity, mixing internal energies and environmental effects indiscriminately.

It is not surprising that associating with the protein is entropically unfavorable, since the protein is a rigid impenetrable object on the lipid isomerization time scale; the difference in time scales means that the lipid must adjust to the protein shape, with the result that certain fluctuations are suppressed. The 
enormous flexibility and energetic degeneracy of DHA allows it to accommodate this gracefully, as indicated by previous simulations which showed that even tightly bound DHA chains isomerize and reorient at roughly the same rate as in bulk lipid. ${ }^{13}$ By contrast, the more constrained STEA chain has a much smaller number of states which can be populated in the presence of the protein. Perhaps, a cell membrane could reduce this entropic penalty by pre-ordering the chains, for instance by including cholesterol in the membranes; this would then work to increase the stability and solubility of membrane proteins.

This work suggests a number of questions to be answered by future calculations and experiments. First, how general are the effects observed? The present simulations consider only one membrane protein in a specific (if biologically relevant) membrane environment, one containing a mixture of two headgroup types and cholesterol. Given that cholesterol is known to preferentially associate with saturated chains, ${ }^{21,22}$ it is worth considering how the results might differ if the membrane composition were varied. Second, is the association between DHA and rhodopsin related to the beneficial effects of dietary PUFAs? Given that many GPCRs, including rhodopsin, are found in membranes enriched in PUFAs, this would appear to be a fruitful avenue for future pursuits, especially as structural information becomes available for more GPCRs.

Acknowledgment. We acknowledge the contributions of the Blue Matter team (B. Fitch, R. Germain, A. Rayshubskiy, T.J.C. Ward, M. Eleftheriou, F. Suits, R. Zhou, J. Pitera, W. Swope, and Y. Zhestkov). We would also like to thank Frank Suits for assistance in developing some of the tools used in this analysis. S.E.F. thanks the NSF for support through award MCB-0091508 and the Dreyfus Foundation for support under the Henry Dreyfus Teacher-Scholar program.

Supporting Information Available: Details of the system construction protocol and molecular dynamics simulation condi- tions. This material is available free of charge via the Internet at http://pubs.acs.org.

\section{References and Notes}

(1) Salem N., Jr.; Litman, B.; Kim, H.-Y.; Gawrisch, K. Lipids 2001, $36(1), 1-15$.

(2) Eldho, N. V.; Feller, S. E.; Tristram-Nagle, S.; Polozov, I. V.; Gawrisch, K. J. Am. Chem. Soc. 2003, 125(21), 6409-6421. 422.

(3) Feller, S. E.; Gawrisch, K. Curr. Opin. Struct. Biol. 2005, 15, 416-

(4) Mihailescu, M.; Gawrisch, K. Biophys. J. 2006, 90, L04-6L.

(5) Stillwell, W.; Wassall, S. R. Chem. Phys. Lipids 2003, 126, 1-27.

(6) Brown, M. F.; Deese, A. J.; Dratz, E. A. Methods Enzymol. 1982, $81,709-728$.

(7) Deese, A. J.; Dratz, E. A.; Brown, M. F. FEBS Lett. 1981, 124 , 93-99.

(8) Wiedmann, T. S.; Pates, R. D.; Beach, J. M.; Salmon, A.; Brown, M. F. Biochemistry 1988, 27, 6469-6474.

(9) Brown, M. F. Chem. Phys. Lipids 1994, 73, 159-180.

(10) Mitchell, D. C.; Niu, S.-L.; Litman, B. J. J. Biol. Chem. 2001, 276(46), 42801-42806.

(11) Feller, S. E.; Gawrisch, K.; Woolf, T. B. J. Am. Chem. Soc. 2003, 125(15), 4434-4435.

(12) Grossfield, A., Feller, S. E., Pitman, M. C. Proc. Natl. Acad. Sci. U.S.A. 2006, 103, 4888-4893.

(13) Pitman, M. C.; Grossfield, A.; Suits, F.; Feller, S. E. J. Am. Chem. Soc. 2005, 127, 4576-4577.

(14) Fattal, D. R.; Ben-Shaul, A. Physica A 1995, 220, 192-216.

(15) Qian, H. Phys. Rev. E 2001, 63, 042103.

(16) Bohinc, K.; Kralj-Iglic, V.; May, S. J. Chem. Phys. 2003, 119 $7435-7444$

(17) Botelho, A. V.; Gibson, N. J.; Thurmond, R. L.; Wang, Y.; Brown, M. F. Biochem. 2002, 41, 6354-6368.

(18) Harries, D.; Ben-Shaul, A. J. Chem. Phys. 1997, 106, 1609-1619.

(19) Jensen, M. O.; Mouritsen, O. G. Biochim. Biophys. Acta 2004, 1666, 205-226.

(20) Meraldi, J.-P.; Schlitter, J. Biochim. Biophys. Acta 1981, 645, 183192.

(21) Wassall, S. R.; Brzustowicz, M. R.; Shaikh, S. R.; Cherezon, V.; Caffrey, M.; Stillwell, W. Chem. Phys. Lipids 2004, 132, 79-88.

(22) Pitman, M.; Suits, F.; MacKerell, A. D., Jr.; Feller, S. E. Biochemistry 2004, 43, 15318-15328. 